\title{
Influence of boundary slip layout on the hydrodynamic performance of partially textured journal bearing by CFD method
}

\author{
Mohammad Tauviqirrahman ${ }^{1, *}$, Muchammad ${ }^{1,2}$, Rizky Amanullah Akbar ${ }^{1}$, and Jamari ${ }^{1}$ \\ ${ }^{1}$ Laboratory for Engineering Design and Tribology, Department of Mechanical Engineering, \\ University of Diponegoro, 50275 Semarang, Indonesia \\ ${ }^{2}$ Laboratory for Surface Technology and Tribology, Faculty of Engineering Technology, University \\ of Twente, Drienerloolan 5, Postbus 217, 7500 AE, Enschede, The Netherlands
}

\begin{abstract}
Modification of the lubricated surface by combining the boundary slip and the texturing has proven to enhance the hydrodynamic performance of bearing. The present work explores the combined effect of boundary slip and texturing varying slip layout on the performance of journal bearing using computational fluid dynamic (CFD) approach. The effect of eccentricity ratio is also of particular interest. The results show that when the boundary slip is located on all edges of textured surface, more enhanced load support by $4 \%$ is obtained in comparison with classical (no-slip) textured one. However, in general the results also indicate that introducing the boundary slip with different layouts on partially textured surface does not much affect the hydrodynamic pressure as well as the load support; therefore, the slip-textured bearing shows similar and closer trend to the no-slip textured one. In addition, it is also found that the effect of eccentricity ratio is more influential than the boundary slip in terms of load support.
\end{abstract}

\section{Introduction}

Surface textures (such as dimples, grooves, etc.) are patterns manufactured on the surfaces of tribological components to improve their performance. The capability of generating additional load support is found to become the most dominant feature of surface texturing. However, the majority of analytical analysis rely on the solution of the Reynolds equation which is often questionable due to the absence of the inertia and body force terms.

Dobrica and Fillon [1] highlighted that the validity of Reynolds equation for texturing slider contacts depends on the Reynolds number and the texture's aspect ratio. Later, considering thermohydrodynamic effect, Cupillard et al. [2] explored the performance of a partially textured slider bearing with a temperature-dependent lubricant using a commercial CFD software. Meng et al. [3] investigated the tribological performance for the bearing with the compound dimple using fluid-structure-interaction FSI approach.

\footnotetext{
Corresponding author: mtauviq99@yahoo.com
} 
In addition to surface texturing, the effect of the boundary slip on the lubrication performance of the journal bearing comes to be an important issue. Several researchers such as [4-7] have explored the behavior of the sliding contact using an optimized boundary slip with respect to load support. The results of all these investigations showed the existence of a lifting force (load support) even there is no wedge effect (two parallel sliding surfaces) using such a boundary slip condition.

Recently, the idea to combine the boundary slip and texturing effects has increased the researches on tribological performance of slip-textured bearing due to the advances found in their performance. Rao et al. [8] explored the combined effects of texturing and boundary slip on the enhancement in load support both in slider and journal bearing. For the case of slider bearing, extensive work has been performed out by Tauviqirrahman et al. $[7,9]$. It was found that compared to a no-slip textured surface, the load support of the combined slip/textured configuration can be enhanced by around $300 \%$ using the optimized slip parameters [7]. In addition, introducing slip at the leading edge of the single-textured bearing is more efficient compared to the pattern in which the slip is placed on the all edges of textured zone in terms of the load support [9]. In recent publication, Lin et al. [10] investigated the influence of large-area slip/texture surface on journal bearing considering cavitation. They claimed that slip/texture pattern would not affect the pressure and load support when it locates at cavitation zone.

In this paper, a configuration of partially textured journal bearing is used in order to assess the benefits from the existence of boundary slip varying its layouts on textured area. It should be noted that in this research the textured region of bearing is located at nocavitation region (i.e. convergent area). Regarding with the wedge effect, the eccentricity ratio is also examined for all textured configurations using computational fluid dynamics (CFD) method based on Navier-Stokes equation.

\section{Methodology}

\subsection{Governing equations}

In the present study, the lubrication problem is solved by the Navier-Stokes equation and continuity equations. The Navier-Stokes (N-S) equations are solved over the domain using a finite-volume method with the commercial CFD software package FLUENT ${ }^{\circledR}$. The Navier-Stokes and the continuity equations can be expressed, respectively.

$$
\rho \frac{D u_{i}}{D t}=-\frac{\partial p}{\partial x_{i}}+\rho G_{i}+\frac{\partial}{\partial x_{j}}\left[2 \eta e_{i j}-\frac{2}{3} \eta\left(\nabla \cdot u_{i}\right) \delta i j\right]
$$

With the application of sliding surfaces in very narrow-gap conditions and the availability of hydrophobic materials, the classical no-slippage boundary condition can be broken down. When lubricant slips along a solid-liquid interface, the slip length $\beta$ is generally used to address the relation between slip velocity and surface shear rate, i.e.

$$
u_{s}=\left.\beta \frac{\partial u}{\partial z}\right|_{\text {surface }}
$$

where $u_{s}$ indicates the streamwise slip velocity at the hydrophobic surface, $\beta$ denotes the slip length and $\partial u / \partial z$ is the surface shear rate. It is usually postulated that a large value of 
$\beta$ implies greater slip. In the present study, the slip length of a hydrophobic surface is assumed as uniform in space and set to $100 \mu \mathrm{m}$ based on published work [11].

In order to model the Navier slip behavior (Eq. 3) in FLUENT, it is necessary to make an additional subroutine to enhance FLUENT's capability and customize its feature for a lubrication modelling analysis. This subroutine known as user-defined-function (UDF). In this way the Navier slip boundary condition can be applied to surfaces of the lubricated sliding contact.

In the CFD approach, meshing of the computational domain is needed in order to solve the continuity and momentum equations over each grid cell. Thus, in the present work the high-resolution scheme in FLUENT is used to discretize. It should be noted that the meshing process for the smooth and textured surfaces has been checked to ensure grid independent results. For the textured surfaces the number of elements of the grid is higher than in the smooth case.

\subsection{CFD model}

Figure 1 depicts the schematic of the partially slip-textured journal bearing varying the boundary slip layouts. The surface texture is positioned in the convergent region (122 $170^{\circ}$ ). For all following computations, for representing partially textured bearing, ten grooves are used. For each groove, the ratio of groove depth over minimum film thickness of 4.4 is implemented. The main parameters of journal bearing studied here is shown in Table 1 in more detail.

In the present study, three patterns of slip layout are introduced. "Pattern 1" is referred to the textured pattern in which the slip is located on the valley of groove cell (Figure. 1a), while "Pattern 2" denotes to the pattern in which the slip is introduced in the hill of groove cell (Figure. 1b). For the slip located in the hill and valley of textured surface, the term "Pattern 3" is used (Figure. 1c). In this study, the moving displacement of journal shaft (namely, the eccentricity ratio $\varepsilon$ ) is the main quantity of interest as an unknown variable.

Table 1. The main parameters of journal bearing.

\begin{tabular}{|c|c|c|}
\hline Parameter & Data Setting & Unit \\
\hline Total length, $L_{z}$ & 133 & $\mathrm{~mm}$ \\
\hline Journal radius, $r$ & 50 & $\mathrm{~mm}$ \\
\hline Housing radius, $R$ & 50.15 & $\mathrm{~mm}$ \\
\hline Radial clearance, $C$ & 0.06 & $\mathrm{~mm}$ \\
\hline Eccentricity ratio, $\varepsilon$ & $0.2 ; 0.5 ; 0.8$ & - \\
\hline Shaft rotation, $U$ & 2,405 & $\mathrm{~m} / \mathrm{s}$ \\
\hline Lubricant density, $\rho$ & 840 & $\mathrm{~kg} / \mathrm{m}^{3}$ \\
\hline Lubricant viscosity, $\mu$ & 0.0127 & $\mathrm{~Pa}-\mathrm{s}$ \\
\hline$W_{\text {texture }}$ & 4 & $\mathrm{~mm}$ \\
\hline$W_{\text {gap }}$ & 0.225 & $\mathrm{~mm}$ \\
\hline
\end{tabular}



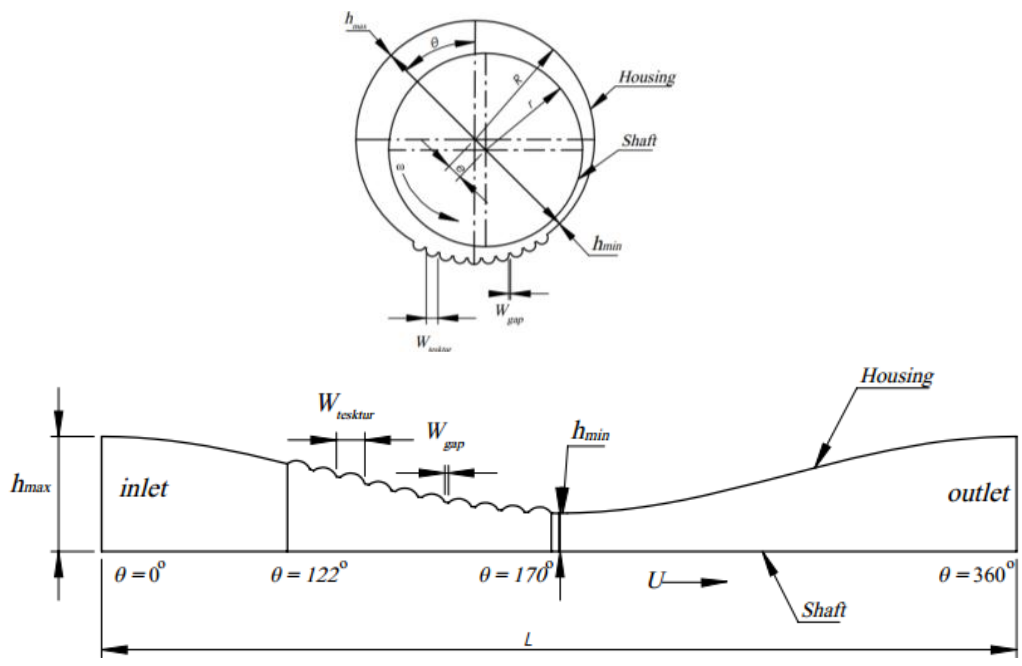

(a)
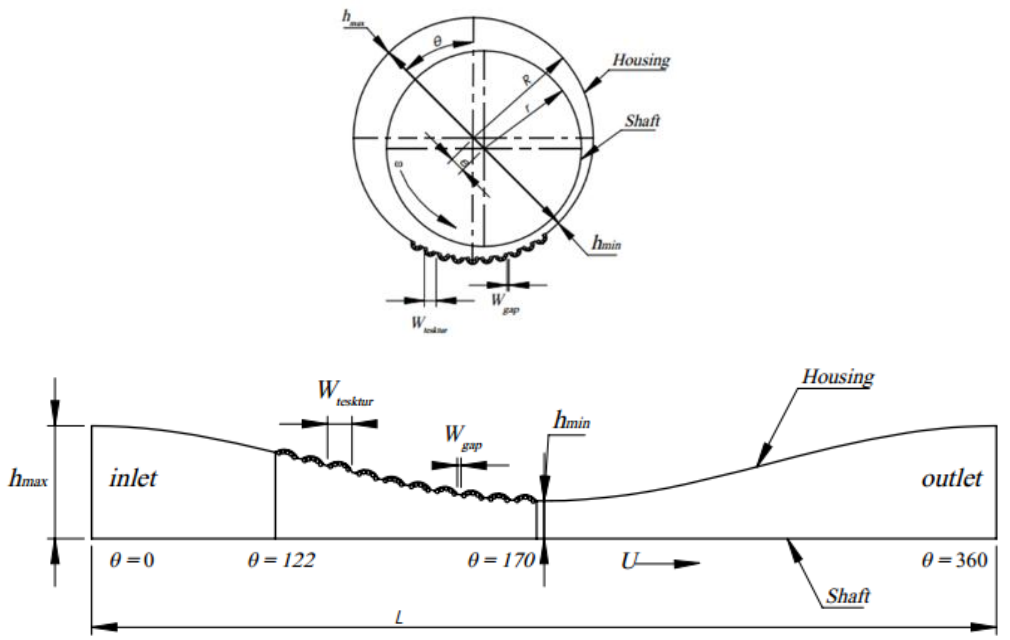

(b) 

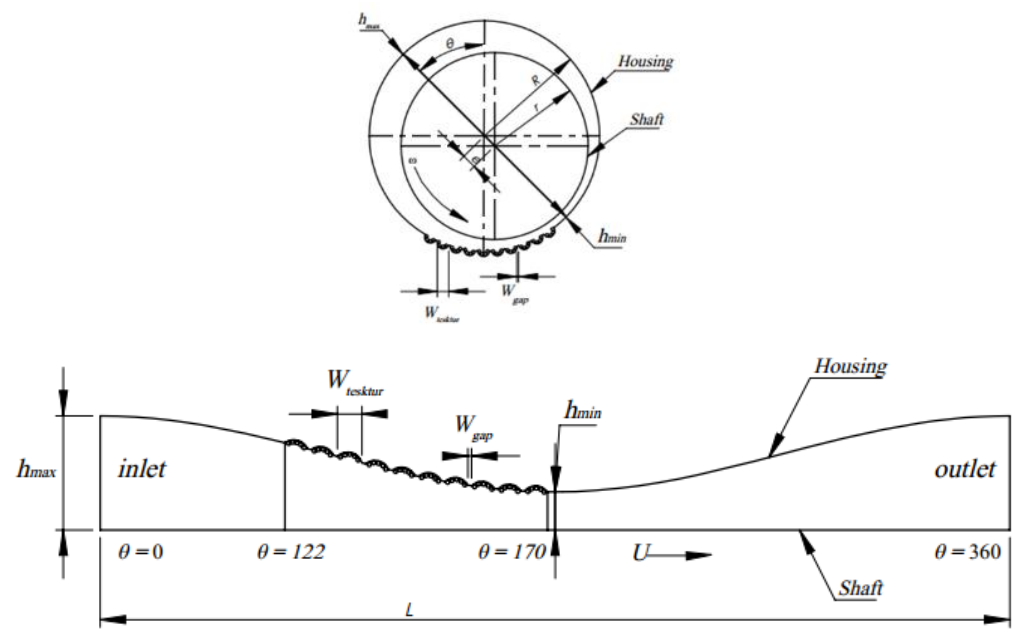

(c)

Fig. 1. Schematic of partially textured journal bearing with different slip layouts: (a) slip is located at the valley of the groove cell (Pattern a), (b) slip is located at the hill of the groove cell (Pattern b), (c) slip is located at the valley and hill of the groove cell (Pattern c).

\section{Results and discussions}

Figure 2 depicts the hydrodynamic pressure over the lubricated surface for four cases in the case of eccentricity ratio of 0.2. In this section, three slip-textured patterns (shown in Figure 1) are compared to the classical journal bearing with no-slip condition.

It can be seen from Figure 2 that for all patterns, the pressure profiles have the similar trend, i.e. the pressure has two peaks of pressure. From the physical point of view, it seems that the texturing gives an effect in altering the hydrodynamic pressure. Based on this figure, it seems that Pattern c gives the highest peak pressure than others. This is as expected because when more boundary slip is placed on surface, more pressure will be generated. As a consequence, Pattern c produces the highest load support and this pattern increases it by $4 \%$ compared to no-slip textured bearing.

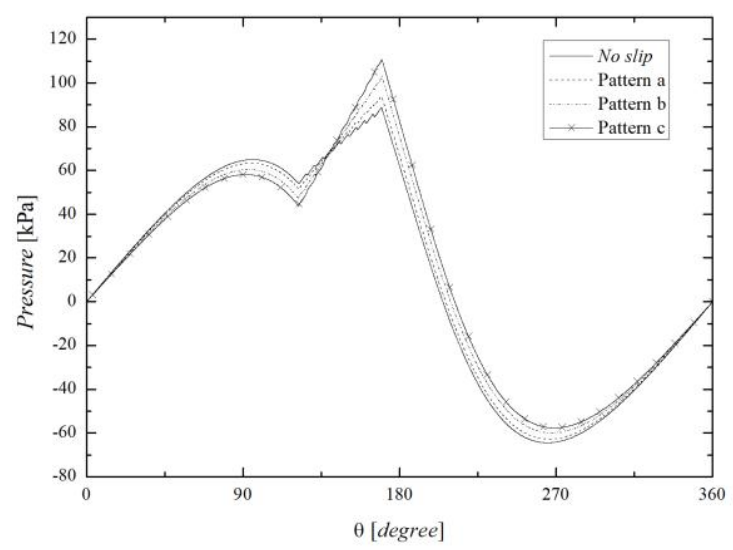

Fig. 2. Hydrodynamic pressure for different patterns for eccentricity ratio $\varepsilon$ of 0.2 . 
Figure 3 shows the hydrodynamic pressure distribution for the case of eccentricity ratio $\varepsilon$ of 0.5 . Compared to pressure profile shown in Figure 2, it can be observed that the wedge effect due to increased eccentricity ratio gives more hydrodynamic pressure for all patterns. For example, the peak pressure for Pattern c in the case of $\varepsilon$ of 0.5 is $347 \mathrm{kN}$, while for $\varepsilon$ of 0.2 the pressure is just $110 \mathrm{kN}$. It means there is an increase in peak pressure up to $200 \%$ due to the wedge effect. Again, the highest load support is obtained when Pattern $\mathrm{c}$ is used.

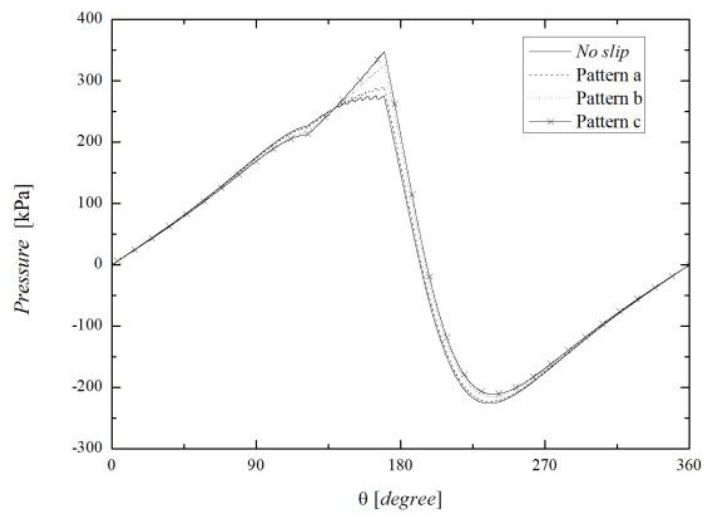

Fig. 3. Hydrodynamic pressure for different patterns for eccentricity ratio $\varepsilon$ of 0.5 .

The distribution of hydrodynamic pressure for different slip-textured patterns for higher eccentricity ratio (i.e. 0.8 in this case) is reflected in Figure 4. Based on Figure 4, there are two specific features. Firstly, for all patterns the pressure trend of increase-then-decrease is observed. Secondly, three patterns considered here generate the same profile with the classical bearing. It indicates that the wedge effect has more significant role in creating the pressure distribution than slip effect. On the other words, the slip effect can be broken down if the eccentricity ratio is relatively high. This result is comparable with the published work [7] in the case of slider bearing.

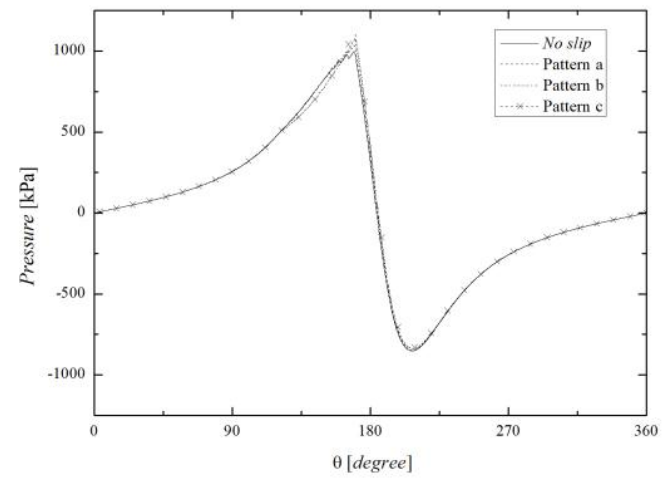

Fig. 4. Hydrodynamic pressure for different patterns for eccentricity ratio of 0.8 . 


\section{Conclusions}

In the present work, based on CFD approach the influence of boundary slip layout placed on textured surface on the hydrodynamic performance of journal bearing was investigated. The effect of eccentricity ratio was also of particular interest. Based on simulation results discussed earlier, the main conclusions can be drawn as follows:

1. When the boundary slip is placed on all edges of textured surface, the improved hydrodynamic pressure as well as the load support is highlighted. However, the increase in load support is not significant, only up to $4 \%$.

2. The more boundary slip effect is observed in partially textured journal bearing when the lower eccentricity ratio is used.

3. The higher the eccentricity ratio used, the more hydrodynamic pressure can be generated, and thus this leads to enhanced load support with or without the presence of boundary slip.

\section{References}

1. M. Dobrica, M. Fillon, Proc. Inst. Mech. Eng. Part J : J. Eng. Tribol. 223, 69 (2009)

2. S. Cupillard, S. Glavatskih, M.J. Cervantes, Tribol. Int. 42, 1487 (2009)

3. F. Meng, L. Zhang, Y. Liu, T. Li, Tribol. Int. 91, 99 (2015)

4. R.F. Salant, A.E. Fortier, Tribol. Trans. 47, 328 (2004)

5. C.W. Wu, G.J. Ma, P. Zhou, ASME J. Tribol. 128, 904 (2006)

6. G.J. Ma, C.W. Wu, Z. Zhou, Tribol. Int. 40, 1056 (2007)

7. M. Tauviqirrahman, R. Ismail, J. Jamari, D.J. Schipper, Acta Mech. 224, 365 (2013)

8. T.V.V.L.N. Rao, A.M.A. Rani, T. Nagarajan, F.M. Hashim, Tribol. Int. 56, 121 (2012)

9. M. Tauviqirrahman, W.K. Ajie, E. Yohana, M. Muchammad, J. Jamari, Int. J. Eng. Tech. 8(2), 913 (2016)

10. Q. Lin, Z. Wei, N. Wang, W. Chen, Ind. Lubr. Tribol. 67, 216 (2015)

11. J.H. Choo, R.P. Glovnea, A.K. Forrest, H.A. Spikes, ASME J. Tribol.129, 611 (2007) 